\title{
Beam Transport in a Quadrupole Electrostatic System
}

\author{
J. J. Barroso ${ }^{1}$ and M. O. Terra ${ }^{2}$ \\ ${ }^{1}$ Instituto Nacional de Pesquisas Espaciais - INPE, Laboratório Associado de Plasma, \\ 12245-970 São José dos Campos, SP, Brazil \\ ${ }^{2}$ Intituto Tecnológico de Aeronáutica - ITA, Departamento de Matemática, \\ Praça Marechal Eduardo Gomes, 50, 12228-900, São José dos Campos, SP, Brazil
}

Received on 16 January, 2004; revised version received on 30 April, 2004

\begin{abstract}
Electron beam transport through a quadrupole electrostatic system is investigated by particle-in-cell simulation in the present work, where - at the advantage of easier experimental implementation - the analogous parabolic electrostatic potential replaces the usual neutralizing ion background of bounded plasma systems. Looking at the maximum transported current and the dynamical behavior dependence on the electron beam injection energy, we have found that for a partial neutralizing electrostatic potential (i) the transmitted current significantly increases in relation to other electrostatic devices, due mainly to two-dimensional effects, (ii) the occurrence of stable static solutions with the typical profile of unstable static solutions of the classical Pierce diode, and (iii) a new bifurcation sequence of the steady-state solutions, at which periodic virtual cathode oscillations turn into intermittent spiking oscillations, which ultimately evolve to stable oscillations when increasing the input energy of the injected electron beam.
\end{abstract}

\section{Introduction}

Research on bounded plasma systems has shown that the presence of a controlled amount of plasma inside a microwave tube can significantly improve the characteristics beyond what is achieved in evacuated devices [1]. In particular, the presence of plasma can increase the operating frequency and power handling capability in microwave tubes by allowing the electron beam to propagate a distance far greater than in a vacuum tube. This idea had been set forward by Pierce in the 1940s [2] upon examining a planar diode where a background of massive ions is included to neutralize the space charge of a electron stream, thereby increasing the maximum stable current that can be transmitted across the interelectrode region. Pierce considered a one-dimensional flow of a neutralized electron beam bounded by two planar surfaces held at the same potential $\Phi(0)=\Phi(d)$, where $d$ denotes the system length. By assuming that a monoenergetic electron beam with velocity $v_{0}=v(0)$ and unperturbed charge density $\rho_{0}=\rho(0)$, equal to the ion background's, is injected at the cathode he found that this idealized system contains a purely growing instability for the single control parameter $\alpha=\omega_{p} d / v_{0}$ slightly greater than $\pi$, where $\omega_{p}$ is the electron plasma frequency. Subsequent works [3]-[8] elucidating the nature of this instability have found, through linear stability analysis, time growing nonoscillatory solutions for $(2 n-1) \pi<\alpha<$ $2 n \pi,(n=1,2,3, \ldots)$, growing oscillatory solutions for $2 n \pi<\alpha<\left(2 n+1-\epsilon_{n}\right) \pi$, and damped solutions for $\left(2 n+1-\epsilon_{n}\right) \pi<\alpha<(2 n+1) \pi$, with $0<\epsilon_{n} \ll 1$, such that the stability character of the linear oscillatory solutions alternates as the parameter $\alpha$ increases at steps of $\pi$. Attrac- ting considerable interest, the classical Pierce diode is the simplest model of distributed microwave electronic systems which is able to present a rich and complex set of nonlinear dynamics.

Exploring the idealized Pierce diode with a view to practical realization, here we investigate the two-dimensional electron flow in a quadrupole electrostatic system emulating the function of the uniform background of stationary ions, which confined by two equipotential surfaces, produces a parabolic potential profile along the electron stream. While preserving the basic features of a plasma-filled diode, the vacuum tetrode discussed here brings in the additional advantage of a much simpler experimental implementation. Through particle-in-cell simulation, we show that the quadrupole system operates in three regimes characterized by the electron beam injection energy $T_{0}$ at fixed potentials $+V_{0}$ and $-V_{0}$ symmetrically applied on the four electrodes. In fact, regular oscillatory behavior accompanied by the formation of virtual cathode reflecting a fraction of the electron beam back to the cathode takes place at low values of $T_{0}$; this is a stable regime in which the self-consistent potential corresponds to an unstable equilibrium in the classical Pierce diode with $\alpha=2.5 \pi$. When $T_{0}$ is increased to reach a threshold value, intermittent oscillations appear accompanied by virtual cathode discharges. And increasing $T_{0}$ even further, a stable static solution is ultimately reached.

In the following, section 2 describes the quadrupole system and PIC simulation parameters. Then, results of the numerical experiments are given and discussed in section 3 , while the last section contains the conclusions of the present work. 


\section{Description of the Quadrupole Sys- tem}

As shown in Fig. 1, the quadrupole fields have two planes of symmetry. Quadrupole fields are a special case of cylindrical multipole fields ( 2 n poles) which satisfy $(\nabla \cdot \mathbf{E}=\mathbf{0}$ and $\nabla \times \mathbf{B}=\mathbf{0}$, where the variation of the radial field component is proportional to $f(z) r^{n-1} \cos [2(n-1) \theta]$. Thus a pure electric quadrupole field $(\mathrm{n}=2)$ in Cartesian coordinates is expressed as $E_{x}=-E_{0}(x / a)$ and $E_{y}=E_{0}(y / a)$. Such a two-dimensional field is produced by four (hyperbolashaped) electrodes symmetrically placed with the center $(0,0)$ equidistant from the four vertices and so with the asymptotes at right angles (Fig. 1). If potentials $V_{0}$ and $-V_{0}$ are applied, the potential distribution in the space between electrodes is given by

$$
V(x, y)=\frac{V_{0}}{a^{2}}\left(x^{2}-y^{2}\right),
$$

which relates the field amplitude $E_{0}$ through $E_{0}=2 V_{0} / a$. Therefore, the electrostatic potential on the $y=0$ plane is parabolic as the one produced by the background of stationary ions in the classical Pierce diode. In fact, a uniform ion background of charge density $\rho_{0}$ confined by two shortcircuited surfaces separated apart by the interelectrode distance $d=2 a$ produces a parabolic potential distribution given by $\Phi(x)=-\left(\rho_{0} / \epsilon_{0}\right) / 2(x-a)^{2}$. In practice, and in the present work, the electric quadrupole potential in (1) is approximated with good accuracy by using four cylindrical rods with radius $a$, rather than electrodes with hyperbolic shapes.

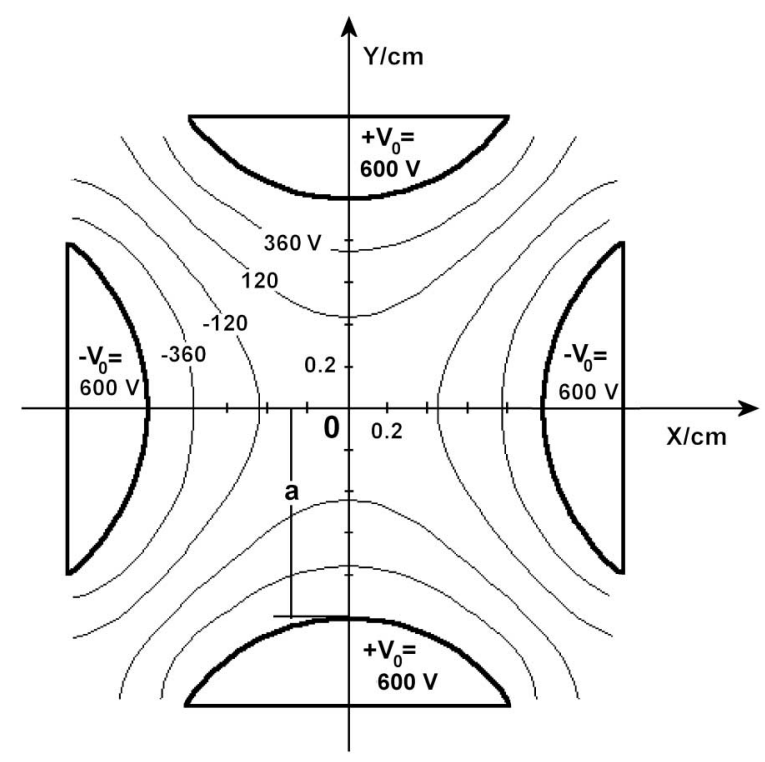

Figure 1. Electrodes and equipotential lines in an electrostatic quadrupole.

In the particle-in-cell (PIC) simulations that follow, a sheet electron beam (1-mm thick and confined by a $5-\mathrm{kG}$ magnetic field) is injected at a given initial kinetic energy from the left side and collected by the electrode on the right (Fig. 2). Sufficiently thin so that a uniform potential develops across its transverse direction, the sheet beam is centered on the bisecting plane $y=0.7 \mathrm{~cm}$ of the twodimensional system with the third dimension $(z)$ being infinite. By using the PIC code KARAT [9], the physical system is run at 0.5 ps time step on a spatial grid of square cell $0.5 \mathrm{~mm}$ size with over 3000 macroparticles representing the beam electrons.

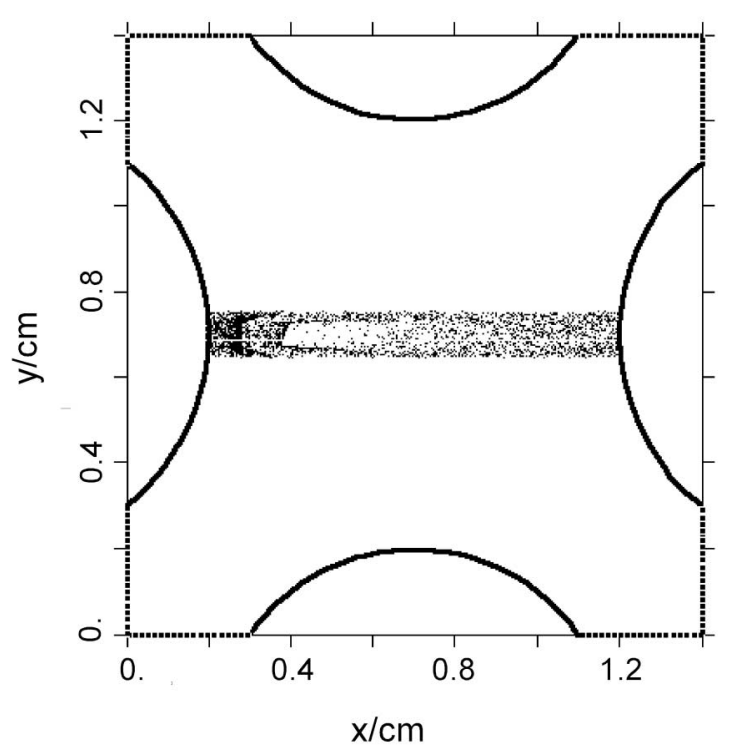

Figure 2. Electrostatic quadrupole at the potentials $+600 \mathrm{~V}$ and $-600 \mathrm{~V}$ traversed by a sheet electron beam injected with initial kinetic energy of $220 \mathrm{eV}$ showing virtual cathode formation. Note that the coordinate system's origin here is defined differently in relation to the previous figure (dislocated to the left lower corner of the system).

\section{PIC Simulation Results}

In the following numerical experiments to investigating the dynamical behavior of the quadrupole system, the electron beam injection energy $T_{0}$ was varied while keeping constant the particle density at the injection plane, the electrode distance, and the applied potential $V_{0}$. At the fixed observation point defined by $x=0 \mathrm{~cm}$ and $y=0.7 \mathrm{~cm}$, Figs. 3 and 4 show the electric field $E_{0}$ as a function of time for a set of eight $T_{0}$ values, increasing from $150 \mathrm{eV}$ to $230 \mathrm{eV}$. Two bifurcations are noticed, as reported below. In the $150-\mathrm{eV}$ time history, a periodic spiking oscillation is observed. Virtual cathode formation occurs in the system and one virtual cathode discharge reaches the left electrode regularly at each maximum of $E_{0}$. By increasing $T_{0}$, intermittent spiking sequences replace the regular ones, and then at about 223.3 eV such aperiodic oscillations turn into a stable static solution, shown in Fig. 4 for $T_{0}=225 \mathrm{eV}$ and $230 \mathrm{eV}$. The 212 and $215 \mathrm{eV}$ time histories correspond to main frequencies of intermediate value as can be seen in Fig. 5. At $T_{0}=221$, 222 and $223 \mathrm{eV}$, intermittency becomes evident in the time series, for which the corresponding smeared spectra (Fig. 6) are unlikely to define a main oscillation frequency. 


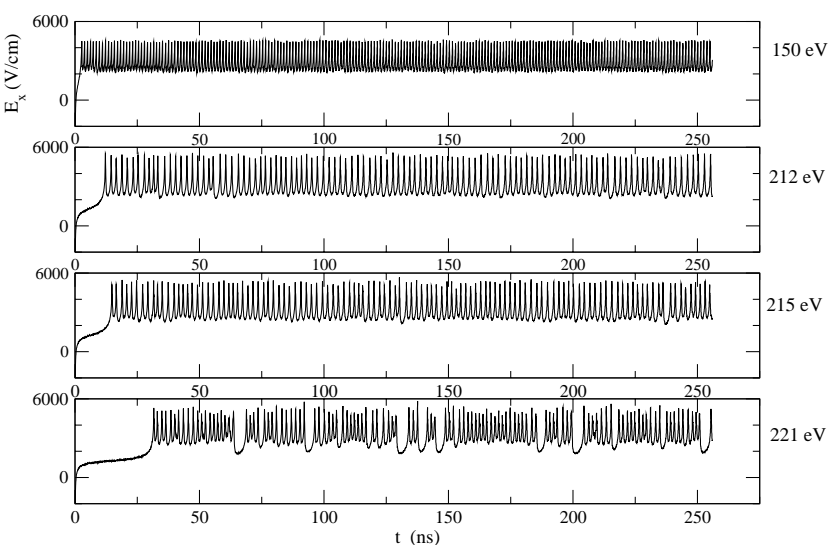

Figure 3. Time history of electric field, $E_{0}$, at $x=0 \mathrm{~cm}$ and $y=0.7 \mathrm{~cm}$ for increasing values of the electron beam injection energy $T_{0}$, presenting a periodic-intermittent oscillation transition.

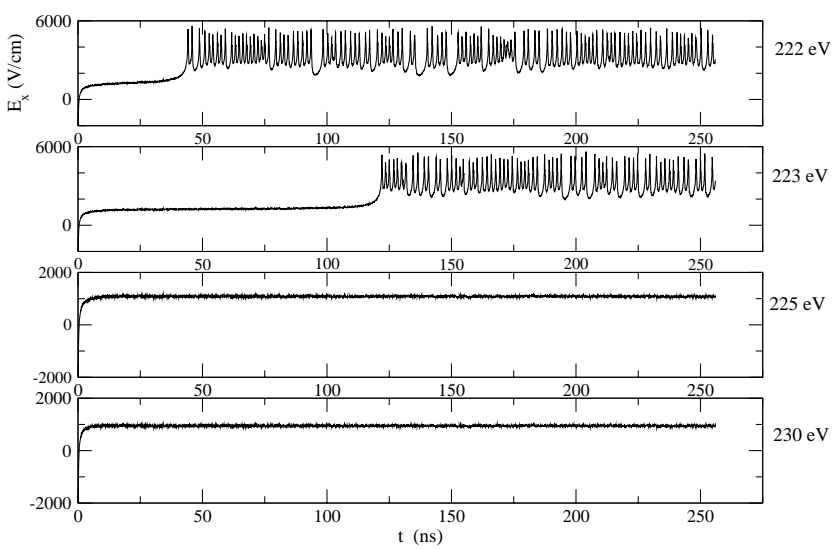

Figure 4. Time history of electric field $E_{0}$ at four different values of the beam injection energy $T_{0}$, presenting intermittency-stationary state bifurcation.

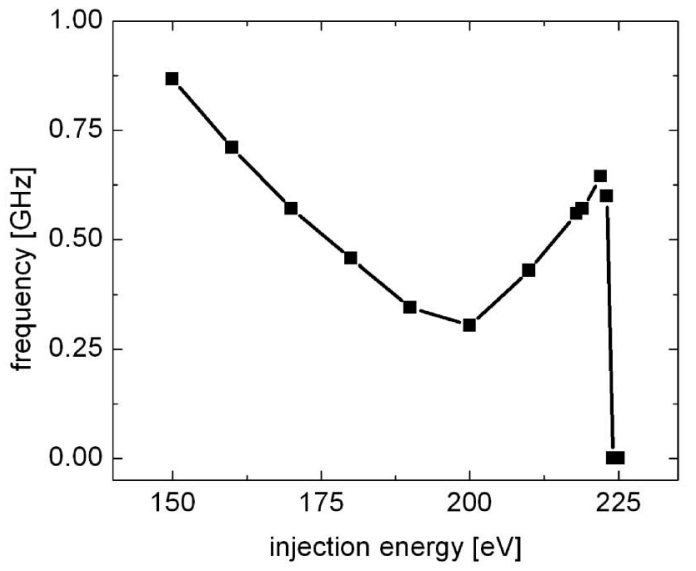

Figure 5. Main oscillation frequency as a function of the injection energy $T_{0}$.
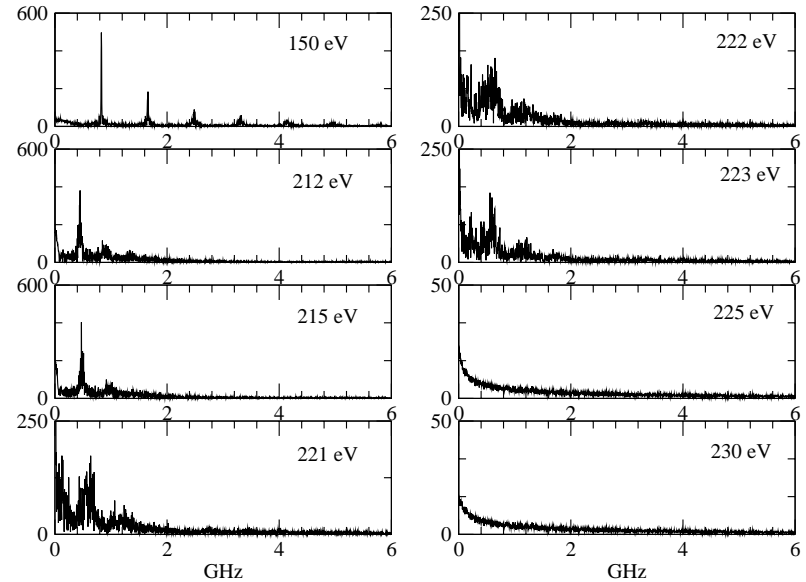

Figure 6. Fourier transform of time series in Figs. 3 and 4.

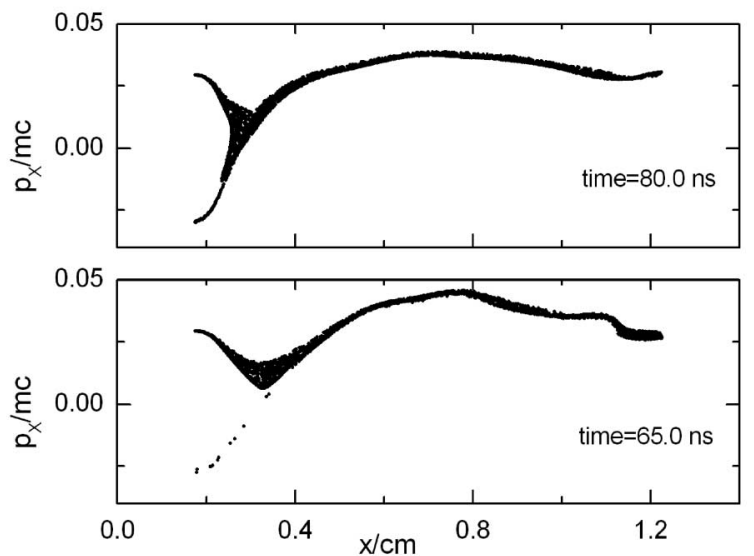

Figure 7. Snapshots of axial momentum at $T_{0}=221 \mathrm{eV}$ for two instants of time: $t=65 \mathrm{~ns}$ and $t=80 \mathrm{~ns}$, respectively a local minimum and a local maximum (showing a virtual cathode discharge) of the $E_{0}$ time history plot.

In Fig. 6, the Fourier transforms of time histories of Figs. 3 and 4 are presented. The three distinct regimes and the two corresponding bifurcations observed in the quadrupole system are indicated by the well-defined-mainfrequency spectra, broad spectra and null frequency spectra. Snapshots of axial momentum at $T_{0}=221 \mathrm{eV}$, corresponding to intermittent regime, are presented for two instants of time in Fig. 7, namely, at $65 \mathrm{ns,} \mathrm{corresponding} \mathrm{to} \mathrm{a} \mathrm{local}$ minimum of $E_{0}$ time history, and at $80 \mathrm{~ns}$, corresponding to a virtual cathode discharge, i.e., a maximum of $E_{0}$ time history. Such phase-space plots illustrate some characteristic patterns of this kind of oscillation.

A remarkable aspect to be mentioned here refers to the spatial dependence of the stable static solution observed in our numerical simulations. The static solution observed for $T_{0}>223.3 \mathrm{eV}$ has positive $E_{0}$ values and a profile similar to the unstable static solutions of the classical Pierce diode, as can be seen in Fig. 8. In this figure the electric potential and axial momentum at $T_{0}=225 \mathrm{eV}, y=0.7 \mathrm{~cm}$ and $t=40 \mathrm{~ns}$ are presented as a function of $x$ coordinate. In the classical Pierce diode [2-8] this kind of equilibrium becomes 
apparent in numerical experiments just with special strategies, as for example constraining some system's quantities for a period of time [5]. This observation suggests that the intensity of the neutralizing field can be used to change the stability properties of time-independent solutions of plasmafilled devices.
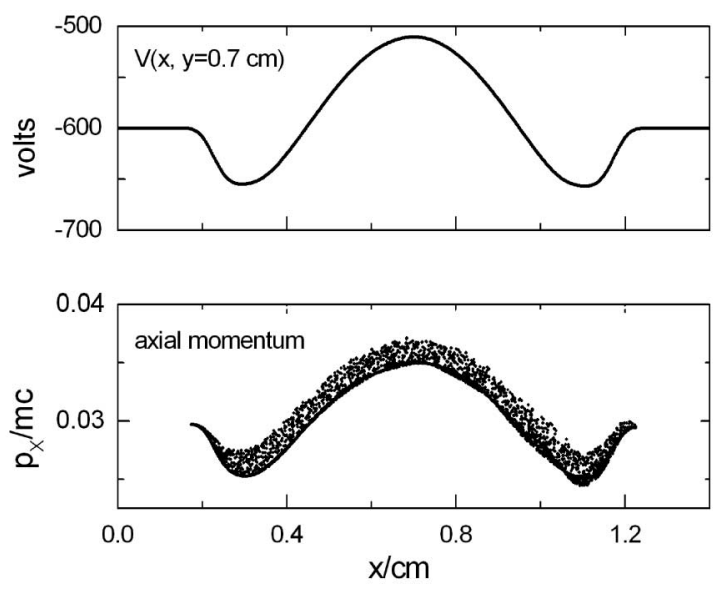

Figure 8. Spatial dependence of potential and axial momentum of observed stable static solution at $T_{0}=225 \mathrm{eV}, t=40 \mathrm{~ns}$ and $y=0.7 \mathrm{~cm}$.

\section{Conclusions}

Beam propagation across the quadrupole is characterized by three operating regimes, which are neatly described by the energy dependence plot of the main frequency as shown in Fig. 5 referring to quadrupole potentials of $+600 \mathrm{~V}$ and $600 \mathrm{~V}$, and beam density of $2.9 \mathrm{~A} / \mathrm{cm}^{2}$ fixed at the injection electrode. In the first regime, oscillation frequency decreases with the initial kinetic energy $T_{0}$ in the range $T_{0}<200 \mathrm{eV}$. Consisting of steady non sinusoidal oscillations with constant period and amplitude, this regime is identified by the scaling relation $f \sim\left(T_{0}\right)^{-3.6}$, which exhibits a much stronger inverse dependence on $T_{0}$ than that for small-amplitude sinusoidal oscillations, characterized by $f \sim\left(T_{0}\right)^{-1 / 4}$, at the corresponding plasma frequency in an electron stream of constant charge density. The second regime develops in the $200 \mathrm{eV}<T_{0}<223 \mathrm{eV}$ range with intermittent oscillations. For $T_{0}$ slightly above $223 \mathrm{eV}$ the oscillations suddenly disappear, and the third regime with static, time-independent solutions is reached. In this context, the oscillation frequency may be interpreted as a measure of the amount of space charge trapped inside the beam, as in the third regime all the injected charge is collected on the exit electrode without virtual cathode formation.

About transmission of stable current, we compare in the table below the quadrupole current, $J_{4 P}$, with the ChildLangmuir and Pierce current densities [2, 4]. Such currents refer to time-independent one-dimensional flow, with the Child-Langmuir current being the maximum current density that can be transported across a short-circuited vacuum diode, with gap spacing $d$,

$$
J_{C L}=\frac{2^{11 / 2}}{9} \epsilon_{0} \sqrt{\frac{e}{m}} \frac{T_{0}^{3 / 2}}{d^{2}},
$$

whereas the Pierce current, $J_{P}$, is the limiting current density that can be transmitted through a short-circuited diode in the presence of neutralizing ions

$$
J_{P}=2^{3 / 2} \pi^{2} \epsilon_{0} \sqrt{\frac{e}{m}} \frac{T_{0}^{3 / 2}}{d^{2}} .
$$

At $T_{0}=225 \mathrm{eV}$ and $d=1.0 \mathrm{~cm}$, we therefore have the current values shown in Table 1.

TABLE 1. Values $\left(\mathrm{A} / \mathrm{cm}^{2}\right)$ for Child-Langmuir, Pierce, and Quadrupole current densities, $J_{4 P}$ at $0 \mathrm{~V}$ and $600 \mathrm{~V}$.

\begin{tabular}{cccc}
\hline$J_{C L}$ & $J_{P}$ & $J_{4 P}(0 \mathrm{~V})$ & $J_{4 P}(600 \mathrm{~V})$ \\
\hline 0.06 & 0.35 & 0.50 & 2.90 \\
\hline
\end{tabular}

Comparing $J_{P}$ with $J_{C L}$ we see that the neutralizing ions increase by a factor of 5.6 the current that can be transmitted through a vacuum diode. The $4 \mathrm{P}$ system, even at $0 \mathrm{~V}$, gives an eight fold increase relative to $J_{C L}$, while polarized at $600 \mathrm{~V}$ it allows an enhancement in current by 48 times. But we would have expected the $4 \mathrm{P}(0 \mathrm{~V})$ and $J_{C L}$ currents to be of the same order. The reason for such difference is accounted for two-dimensional effects [10], because the $J_{C L}$ and $J_{P}$ expressions are strictly valid in one-dimensional geometry, namely, in plane parallel diodes where the beam width (W) is much larger than the gap spacing (L). Accordingly, in the $4 \mathrm{P}$ system the $\mathrm{W} / \mathrm{L}$ ratio is as low as 0.1 . Nevertheless, we see that the maximum allowable current greatly increases with the applied voltage, as when raising the voltage from $0 \mathrm{~V}$ to $600 \mathrm{~V}$, for instance, the current increases by roughly six times, and in this respect lies the main attribute of the quadrupole system studied here.

\section{References}

[1] G. S. Nusinovich, Y. Carmel, T.M. Antonsen, Jr., D. M. Goebel, and J. Santoru, IEEE Trans. Plasma Sci. 26, 628 (1998).

[2] J. R. Pierce, J. Appl. Phys. 15, 721 (1944).

[3] M. O. Terra, J. J. Barroso, and E. E. N. Macau, Physica A 283, 119 (2000).

[4] J. J. Barroso, M. O. Terra, and E. E. N. Macau, International Journal of Bifurcation and Chaos 11, 2579 (2001).

[5] W. S. Lawson, Phys. Fluids B 1, 1493 (1989).

[6] X. Chen, P. A. Lindsay, and J. Zhang, IEEE Trans. Plasma Sci. 28, 462 (2000).

[7] B. B. Godfrey, Phys. Fluids 30, 1553 (1987).

[8] H. Friedel, R. Grauer, and K. H. Spatschek, Phys. Plasmas 5, 3187 (1998).

[9] V. P. Tarakanov, User's Manual for Code KARAT (Springfield, VA: Berkeley Research Associates Inc., 1994).

[10] Y. Y. Lau, Phys. Rev. Lett. 87, 278301 (2001). 